\title{
Gráfico de controle de VMAX para o monitoramento da matriz de covariâncias
}

\author{
Marcela Aparecida Guerreiro Machado FEG/UNESP \\ Maysa Sacramento de Magalhães ENCE/IBGE \\ Antônio Fernando Branco Costa FEG/UNESP
}

\section{RESUMO}

Neste artigo é proposto, para o monitoramento de processos normais bivariados, um gráfico de controle baseado nas variâncias amostrais de duas características de qualidade. Os pontos plotados no gráfico correspondem ao valor da maior variância amostral. 0 gráfico proposto, denominado gráfico de VMAX, tem um desempenho superior ao do gráfico da variância amostral generalizada $|\mathbf{S}|$ e, além disso, tem uma melhor capacidade de diagnóstico, ou seja, com ele é mais fácil identificar a variável que teve sua variabilidade alterada pela ocorrência da causa especial. Quando a amostragem dupla está em uso o gráfico proposto também tem um desempenho superior ao do gráfico de $|\mathbf{S}|$, exceto em alguns casos em que o tamanho da segunda amostra é muito grande.

\section{PALAVRAS-CHAVE}

Gráficos de controle de VMAX, matriz de covariâncias, processos bivariados, amostragem dupla.

\section{The VMAX control chart for monitoring the covariance matrix}

\section{ABSTRACT}

In this paper a control chart based on sample variances from two quality characteristics for monitoring bivariate normal processes is proposed. The points plotted on the chart are the maximum of the values of these two statistics. The proposed chart (VMAX chart) detects process disturbances faster than the generalized variance $|\mathrm{S}|$ chart and has a better diagnostic feature, that is, with the VMAX chart it is easier to relate an out-of-control signal to the variable whose variability has moved away from its in-control state. When the double sampling scheme is used the proposed chart has also a better performance, except in a few cases in which the size of the samples at the second stage is very large.

\section{KEY WORDS}

VMAX control chart, covariance matrix, bivariate processes, double sampling. 


\section{INTRODUÇÃO}

O gráfico de controle tem sido a principal ferramenta estatística utilizada no monitoramento de processos, graças a sua simplicidade operacional. De acordo com os fundamentos de Shewhart, sempre que um ponto é plotado na região de ação do gráfico, deve-se ir à procura de possíveis causas especiais que estejam afetando a qualidade do processo. Entretanto, a simplicidade operacional faz do gráfico de controle de Shewhart uma ferramenta lenta na detecção de pequenas a moderadas alterações no parâmetro do processo que está sendo monitorado. Diante disso, não têm sido poucas as inovações sugeridas ao trabalho original de Shewhart, como por exemplo, gráficos de somas acumuladas (PAGE, 1954; HAWKINS; OLWELL, 1998; REYNOLDS; STOUMBOS, 2005), gráficos de médias móveis ponderadas exponencialmente (LUCAS; SACCUCCI, 1990; DOMANGUE; PATCH, 1991; CHEN et al., 2001; COSTA; RAHIM, 2006), gráficos adaptativos (COSTA, 1994, 1999; De MAGALHÃES et al., 2002, 2006) e gráficos de controle com amostragem dupla (CROASDALE, 1974; DAUDIN, 1992; IRIANTO; SHINOZAKI, 1998; CAROT et al., 2002; HE et al., 2002; HE; GRIGORYAN, 2002, 2006; COSTA et al., 2005a).

A adoção de gráficos de controle com amostragem dupla para o monitoramento do vetor de médias de processos multivariados tem sido objeto de pesquisa recente. He e Grigoryan (2005) avaliaram o desempenho dos gráficos multivariados com amostragem dupla por meio de simulação. Alternativamente, Machado (2006) e Costa e Machado (2007) desenvolveram expressões para obtenção das propriedades dos gráficos bivariados com amostragem dupla.

Assim como é importante monitorar o vetor de médias de um processo, é também importante monitorar a sua matriz de covariâncias (YEH et al., 2003, 2004, 2005; SURTIHADI et al., 2004; GRIGORYAN; HE, 2005).

O primeiro gráfico de controle utilizado no monitoramento da matriz de covariâncias $\Sigma$ se baseou na estatística obtida do teste da razão de máxima verossimilhança generalizada (ALT, 1985). Para o caso bivariado, Alt (1985) propôs o uso da variância amostral generalizada $|\mathbf{S}|$ para controlar a matriz de covariâncias $\Sigma$. É importante salientar que os testes estatísticos para igualdade de matrizes de covariâncias foram propostos bem antes de serem utilizados no monitoramento de processos. Em Anderson (2003), os leitores interessados encontrarão detalhes destes testes.

Aparisi et al. (1999) estenderam a aplicação da estatística $|\mathbf{S}|$ para o caso em que o número de variáveis do processo sob monitoramento é maior do que dois ( $p>2$ ) e, considerando esta mesma estatística, propuseram um gráfico de controle bivariado com tamanho de amostra variável (APARISI et al., 2001). Grigoryan e He (2005) propuseram o gráfico de controle multivariado com amostragem dupla para o monitoramento da matriz de covariâncias. Uma revisão recente de gráficos de controle para o monitoramento da matriz de covariâncias foi elaborada por Yeh et al. (2006). Chang e Zhang (2007) apresentaram uma metodologia para o monitoramento da variabilidade de processos multivariados autocorrelacionados.

Neste artigo é proposto, para o monitoramento de processos bivariados, um gráfico de controle baseado nas variâncias amostrais de duas características de qualidade. Os pontos plotados no gráfico correspondem ao valor da maior variân-

\section{gráfico de controle tem sido a principal ferramenta} processos, graças a sua simplicidade operacional.

cia. O gráfico proposto, denominado gráfico de VMAX, ou seja, da maior variância, tem um desempenho superior ao do gráfico da variância amostral generalizada $|\mathbf{S}|$. Além do gráfico de VMAX, também é proposto o gráfico de VMAX com amostragem dupla. Este gráfico tem também um desempenho superior ao do gráfico da variância amostral generalizada $|\mathbf{S}|$, exceto em alguns casos em que o tamanho da segunda amostra é muito grande. Nos Apêndices I e II são apresentadas as expressões para a obtenção das propriedades dos gráficos de VMAX e de VMAX com amostragem dupla, respectivamente. A principal vantagem do gráfico de VMAX em relação aos gráficos de controle multivariados é que ele possui uma melhor capacidade de diagnóstico, ou seja, com ele é mais fácil identificar a variável que teve sua variabilidade alterada pela ocorrência de uma causa especial.

\section{GRÁFICOS UNIVARIADOS PARA $O$ MONITORAMENTO DE PROCESSOS MULTIVARIADOS}

Uma dificuldade encontrada ao se lidar com qualquer gráfico de controle multivariado é a interpretação prática de um sinal de fora de controle. Especificamente, não se sabe ao certo qual das variáveis (suponha $p$ variáveis) ou qual subconjunto delas é responsável pelo sinal. Na literatura encontramos muitas maneiras para resolver este problema, desde métodos gráficos até técnicas mais analíticas. A técnica de 
decomposição é uma das de maior sucesso (MASON, YOUNG, 2002). Uma outra alternativa consiste em plotar gráficos univariados para controlar cada característica de qualidade de um processo normal multivariado (SEREL et al., 2000).

Como um exemplo de como controlar diferentes variáveis com gráficos de $S^{2}$, considere duas características de qualidade, representadas pelas variáveis normalmente distribuídas $X$ e $Y$. Primeiramente considere o caso em que as duas características são independentes e monitoradas separadamente por dois gráficos de $S^{2}$ univariados com limites de controle de 4 desvios-padrão. Para amostras de tamanho cinco, cada ponto amostral tem uma probabilidade do erro do tipo I igual a 0,004 (ou seja, $\alpha=0,004$ ) de exceder os limites de controle de 4 desvios-padrão. A probabilidade de que as variâncias amostrais estejam dentro dos limites quando o processo está em controle é igual a $(1-0,004)$ $(1-0,004)$. Então a probabilidade do erro total do tipo I (denotada por $\left.\alpha^{\prime}\right)$, para este caso, é dada por $\alpha^{\prime}=1-(1-0,004)$ $(1-0,004)=1-0,992=0,008$. No caso de $p$ características de qualidade estatisticamente independentes, a probabilidade do erro total do tipo I será dada por,

$$
\alpha^{\prime}=1-(1-\alpha)^{p}
$$

Caso se deseje manter controlada a probabilidade do erro do tipo I para um processo $p$-variado com variáveis aleatórias independentes, a equação (1) pode ser utilizada para calcular a probabilidade do erro do tipo I de cada gráfico (MONTGOMERY, 2004). Conseqüentemente, obtém-se o coeficiente de abertura dos limites de controle de cada gráfico. É importante ressaltar que quando se trabalha com um número grande de variáveis, a probabilidade do erro do tipo I de cada gráfico separadamente é muito pequena e o fator de abertura dos limites de controle é muito grande, reduzindo, assim, a sensibilidade do gráfico de controle em detectar desajustes do processo.

Se as variáveis são dependentes, que é o caso mais comum, a obtenção dos limites de controle de cada gráfico deixa de ser trivial. Para simplificar, considere o caso bivariado em que as variáveis seguem uma distribuição normal e que a matriz de covariâncias com o processo em controle é dada

por $\boldsymbol{\Sigma}_{0}=\left(\begin{array}{cc}\sigma_{0 x}^{2} & \rho \sigma_{0 x} \sigma_{0 y} \\ \rho \sigma_{0 x} \sigma_{0 y} & \sigma_{0 y}^{2}\end{array}\right)$, onde $\rho$ é o coeficiente de correlação entre as variáveis $X$ e Y. Se gráficos de $S^{2}$ univariados são utilizados para controlar cada característica de qualidade, então $\alpha$ ' será dado por:

$$
\alpha^{\prime}=1-\operatorname{Pr}\left[\left(S_{x}^{2}<L C \sigma_{0 x}^{2}\right) \cap\left(S_{y}^{2}<L C \sigma_{0 y}^{2}\right)\right]
$$

Para o cálculo do fator de abertura do limite de controle, $L C$, foi necessário obter a expressão (A1) do Apêndice I.

\section{GRÁFICO DE CONTROLE DA MAIOR VARIÂNCIA (GRÁFICO DE VMAX)}

Nesta seção apresenta-se o gráfico de controle proposto para o monitoramento da matriz de covariâncias $\Sigma$ de um processo bivariado. Supõe-se que o vetor de médias $\mu$ não sofre alterações.

A estatística de monitoramento $\operatorname{VMAX}=\max \left\{S_{x}^{2}, S_{y}^{2}\right\}$ corresponde ao maior valor das variâncias amostrais $S_{x}^{2}=\frac{\sum_{i=1}^{n}\left(X_{i}-\mu_{x}\right)^{2}}{n}$ e $S_{y}^{2}=\frac{\sum_{i=1}^{n}\left(Y_{i}-\mu_{y}\right)^{2}}{n}$, sendo $X$ e $Y$ duas características de qualidade que seguem uma distribuição normal bivariada. Sem perda de generalidade, supõe-se que com o processo em controle a matriz de covariâncias é dada por $\boldsymbol{\Sigma}_{0}=\left(\begin{array}{cc}1 & \sigma_{x y} \\ \sigma_{y x} & 1\end{array}\right)$, sendo $\Sigma_{x y}$ e $\Sigma_{y x}$ as covariâncias entre $X$ e $Y$. Na prática, o mais comum é encontrar variáveis com variâncias diferentes entre si e não necessariamente iguais a 1. Contudo, a análise de sensibilidade apresentada neste trabalho permanece válida, uma vez que o efeito da causa especial, que resulta em aumento da variabilidade, é sempre expresso em unidades das variâncias iniciais de $X$ e $Y$, isto é, variâncias de $X$ e $Y$ estimadas com o processo em controle.

Outro ponto importante sobre a matriz de covariâncias $\boldsymbol{\Sigma}_{0}$ que merece atenção é a escolha do estimador a ser utilizado em sua estimação. De acordo com Djauhari (2005) e, segundo estudos que datam da década de 50 (ANDERSON, 2003), o estimador $|\overline{\mathbf{S}}|$ é viesado, sendo $\overline{\mathbf{S}}$ a média das matrizes de covariâncias amostrais $\mathrm{S}_{i}, i=1,2, \ldots, m$, das $m$ amostras de tamanho $n$ utilizadas na estimação de $\boldsymbol{\Sigma}_{0}$. Segue que $|\overline{\mathbf{S}}| \sim\left\{\left|\boldsymbol{\Sigma}_{0}\right| /[m(n-1)]^{p}\right\} \prod_{k=1}^{p} Z_{k}$, onde $Z_{k}$ são independentes e $Z_{k} \sim \chi_{m(n-1)-k+1}^{2}, k=1,2, \ldots, p$. Para eliminar o viés, o estimador $|\bar{S}|$ deve ser dividido por uma constante $b_{3}$ que é função de $m, n$ e $p$. É interessante mencionar que Djauhari (2005) usou a distribuição normal como aproximação da distribuição do determinante da matriz de covariâncias. Neste artigo, porém, o monitoramento é realizado com base nas variâncias amostrais, as quais têm distribuição de Qui-quadrado.

Analogamente a Surtihadi et al. (2004), assume-se duas possíveis maneiras de uma causa especial alterar a matriz de covariâncias, resultando na matriz $\Sigma_{1}=\left(\begin{array}{cc}a \cdot a & a \cdot b \cdot \sigma_{x y} \\ a \cdot b \cdot \sigma_{y x} & b \cdot b\end{array}\right)$. Uma possibilidade (caso I) supõe que a causa especial afeta somente a variância da variável aleatória $X$, isto é, $a=c$ e $b=$ 1 , ou somente a variância da variável aleatória $Y$, isto é, $b=c$ e $a=1$. A outra possibilidade (caso II) supõe que a causa especial altera tanto a variância de $\mathrm{X}$ como a de $\mathrm{Y}$ e, neste caso, $a=b=\sqrt{c}$, sendo $c$ a magnitude da perturbação $(c>1)$. Em 
ambos os casos, a correlação entre $X$ e $Y$, dada por $\rho=\frac{\sigma_{x y}}{\sigma_{x} \sigma_{y}}$,

não é afetada pela causa especial. Da maneira como $\Sigma_{0}$ foi definida, tem-se que $\Sigma_{0 x}=\Sigma_{0 y}=1$ e, conseqüentemente, $\rho=\sigma_{x y}=\sigma_{y x}$.

Quando o gráfico de VMAX está em uso, amostras de tamanho $n$ são retiradas do processo em intervalos de tempo regulares. Duas características de qualidade $\left(X_{i}, Y_{i}\right)$ das $n$ unidades da amostra são medidas e a estatística VMAX $=\max \left\{S_{x}^{2}, S_{y}^{2}\right\}$ é calculada, sendo $S_{x}^{2}$ e $S_{y}^{2}$ as variâncias amostrais de $\left(X_{i}, Y_{i}\right)$.

Se a estatística VMAX for maior do que o limite de controle $L C$, o gráfico sinaliza um desajuste do processo. Após a ocorrência do sinal, o usuário pode imediatamente examinar as variâncias amostrais de $X$ e $Y$ para descobrir quais variáveis são responsáveis pelo desajuste do processo, ou seja, aquelas cujas variâncias amostrais são maiores que $L C$. Este diagnóstico deve ser feito com cautela, pois uma causa especial pode aumentar a variabilidade de $X$ e $Y$ e, para a amostra que sinaliza, não necessariamente, as variâncias amostrais de $X$ e $Y$ serão maiores que o limite de controle. $O$ limite de controle $L C$ do gráfico de VMAX é obtido utilizando a expressão (A1) do Apêndice I. A Figura 1 apresenta o gráfico proposto.

A eficiência de um gráfico de controle em detectar uma alteração no processo pode ser medida pelo número médio de amostras até o sinal (NMA), ver Costa et al. (2005). Durante o período em controle o $N M A=1 / \alpha$ e é denominado $N M A_{0}$. Quando um processo está sob controle é desejável que o número médio de amostras retiradas do processo desde o início do monitoramento até o sinal $\left(N M A_{0}\right)$ seja grande, de modo a garantir poucos alarmes falsos. Quando um processo está fora de controle é desejável que o número médio de amostras retiradas desde a ocorrência de uma

\section{A eficiência de um gráfico de controle em Adetectar uma alteração no processo pode ser medida pelo número médio de amostras até o sinal (NMA), ver Costa et al. (2005).}

causa especial até o sinal (NMA) seja pequeno, de modo a garantir uma rápida detecção de alterações no processo. No Apêndice I é desenvolvida a expressão para obtenção do NMA para o gráfico de VMAX.

O desempenho do gráfico proposto é comparado com o do gráfico da variância amostral generalizada $|\mathbf{S}|$, onde $\frac{2(n-1) \mid \mathbf{S}^{1 / 2}}{|\Sigma|^{1 / 2}}$ tem distribuição de qui-quadrado com $2 n-4$ graus de liberdade (ALT, 1985). A matriz de covariâncias S é

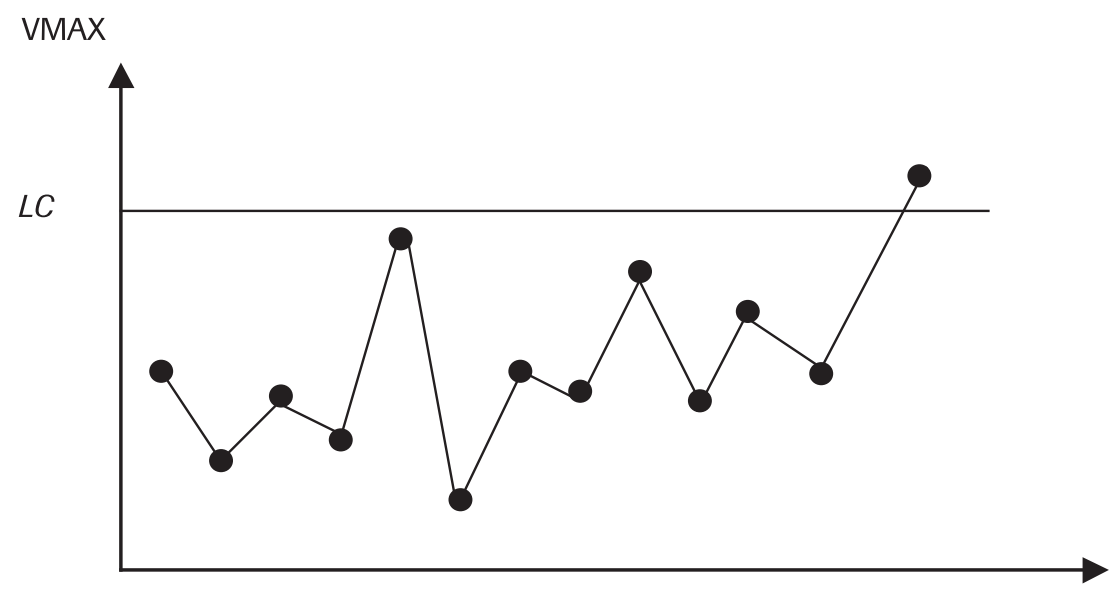

$\begin{array}{lllllllllllll}1 & 2 & 3 & 4 & 5 & 6 & 7 & 8 & 9 & 10 & 11 & 12 & 13\end{array}$

Número da amostra (I)

Figura 1: Gráfico de controle de VMAX. 
dada por $\mathbf{S}=\left[\begin{array}{ll}s_{x x} & s_{x y} \\ s_{y x} & s_{y y}\end{array}\right]$, sendo $s_{x x}$ es $s_{y y}$ as variâncias amostrais de $X$ e $Y$ respectivamente e $s_{x y}$ e $s_{y x}$ as covariâncias amostrais. O limite de controle para o gráfico de $|\mathbf{S}|$ é dado por:

$$
L C=\frac{\left(\chi_{2 n-4, \alpha}^{2}\right)^{2}\left|\Sigma_{0}\right|}{4(n-1)^{2}}
$$

A magnitude da perturbação é dada por $c^{2}$, onde $c^{2}$ é a razão entre $\left|\boldsymbol{\Sigma}_{1}\right|$ e $\left|\boldsymbol{\Sigma}_{0}\right|$. Sendo assim, o gráfico de $|\boldsymbol{S}|$ terá o mesmo poder de detecção para qualquer perturbação na matriz de covariâncias que resulte no mesmo valor de $c^{2}$, independente da causa especial afetar apenas a variável $\mathrm{X}$ ou apenas a variável Y (caso I), ou ambas (caso II). De acordo com a expressão (4) do NMA para o gráfico de $|\mathbf{S}|$, o desempenho deste gráfico independe da correlação (APARISI et al., 2001):

$$
N M A=\frac{1}{\operatorname{Pr}\left(\chi_{2 n-4}^{2} \geq\left(\chi_{2 n-4, \alpha}^{2}\right) / c\right)}
$$

O coeficiente de correlação $\rho$ tem uma certa influência nas propriedades do gráfico de VMAX, ver Tabela 1. Desta forma, escolheu-se um valor de $\rho$ intermediário para a obtenção das propriedades do gráfico de VMAX. De acordo com a Tabela 2 , construída para $\rho=0,5$, o gráfico de VMAX é sempre mais eficiente que o gráfico da variância amostral generalizada $|\mathbf{S}|$. Para uma mesma magnitude de perturbação (mesmo valor de $c^{2}$ ), o gráfico de VMAX é mais sensível se a perturbação afetar apenas uma das variáveis (caso I). Isto se explica pelo fato da estatística de VMAX ser o valor da maior variância amostral. Para o caso I, o gráfico de VMAX tem um desempenho bem superior ao do gráfico de controle proposto por Alt (1985). Os gráficos de controle em todas as tabelas que seguem foram planejados com base em um erro do tipo I da ordem de $0,5 \%$, ou seja, um $N M A_{0}=200,0$.

\section{DESCRIÇÃO DO GRÁFICO DE VMAX COM AMOSTRAGEM DUPLA}

Suponha que o gráfico de controle de VMAX com amostragem dupla seja empregado no monitoramento da matriz de covariâncias $\boldsymbol{\Sigma}$ de duas características de qualidade $\left(X_{i}, Y_{i}\right)$, descritas por uma distribuição normal bivariada.

Similarmente ao gráfico de controle de Shewhart, amostras de tamanho $n=n_{1}+n_{2}$ são retiradas do processo em intervalos de tempo regulares. A amostragem é realizada em dois estágios. No primeiro estágio $n_{1}$ unidades são inspecionadas, as duas características de qualidade são medidas e a estatística $\operatorname{VMAX}_{1}=\max \left\{S_{1 x}^{2}, S_{1 y}^{2}\right\}$ é calculada, sendo $S_{1 x}^{2}$ e $S_{1 y}^{2}$ as variâncias amostrais de $\left(X_{i}, Y_{i}\right)$ da subamostra de tamanho $n_{1}$.

\begin{tabular}{|c|c|c|c|c|c|c|c|c|c|c|c|}
\hline \multirow[b]{4}{*}{$c^{2}$} & \multirow[b]{4}{*}{$L C$} & \multicolumn{5}{|c|}{ caso I } & \multicolumn{5}{|c|}{ caso II } \\
\hline & & \multicolumn{5}{|c|}{$\rho$} & \multicolumn{5}{|c|}{$\rho$} \\
\hline & & 0,0 & 0,1 & 0,5 & 0,7 & 0,9 & 0,0 & 0,1 & 0,5 & 0,7 & 0,9 \\
\hline & & 3,677 & 3,676 & 3,668 & 3,646 & 3,569 & 3,677 & 3,676 & 3,668 & 3,646 & 3,569 \\
\hline 1,0 & & 200,0 & 200,0 & 200,0 & 200,0 & 200,0 & 200,0 & 200,0 & 200,0 & 200,0 & 200,0 \\
\hline 1,1 & & 132,1 & 132,1 & 132,5 & 132,8 & 132,1 & 139,1 & 139,1 & 139,7 & 140,5 & 142,1 \\
\hline 1,2 & & 86,5 & 86,5 & 86,8 & 86,8 & 84,5 & 101,6 & 101,7 & 102,4 & 103,6 & 105,8 \\
\hline 1,3 & & 58,1 & 58,1 & 58,3 & 58,1 & 55,5 & 77,1 & 77,2 & 78,0 & 79,3 & 81,6 \\
\hline 1,4 & & 40,6 & 40,6 & 40,7 & 40,4 & 38,1 & 60,5 & 60,5 & 61,4 & 62,6 & 64,9 \\
\hline 1,5 & & 29,5 & 29,5 & 29,6 & 29,3 & 27,4 & 48,7 & 48,7 & 49,6 & 50,8 & 53,0 \\
\hline 2,0 & & 9,62 & 9,62 & 9,62 & 9,50 & 8,91 & 21,6 & 21,7 & 22,3 & 23,2 & 24,8 \\
\hline 3,0 & & 3,38 & 3,38 & 3,38 & 3,34 & 3,21 & 8,64 & 8,66 & 9,09 & 9,59 & 10,5 \\
\hline 5,0 & & 1,67 & 1,67 & 1,67 & 1,66 & 1,63 & 3,73 & 3,74 & 3,98 & 4,25 & 4,71 \\
\hline
\end{tabular}

Tabela 1: Valores de NMA para o gráfico de $\operatorname{VMAX}(n=5)$. 
Se $\operatorname{VMAX}_{1}$ for menor do que o limite de advertência, $L A$, a amostragem é interrompida. Se $\mathrm{VMAX}_{1}$ for maior do que o limite de controle do primeiro estágio, $L C_{1}$, o gráfico de controle sinaliza um desajuste do processo. Se $L A<\operatorname{VMAX}_{1} \leq L C_{1}$, a amostragem vai para o segundo estágio, onde as $n_{2}$ unidades restantes são inspecionadas e a estatística $\operatorname{VMAX}_{2}=\max \left\{S_{2 x}^{2}, S_{2 y}^{2}\right\}$ é calculada, sendo $S_{2 x}^{2}$ e $S_{2 y}^{2}$ as variâncias amostrais de $\left(X_{i}, Y_{i}\right)$ levando em consideração a amostra de tamanho n. O gráfico também sinaliza um desajuste do processo quando $\operatorname{VMAX}_{2}>L C_{2}$, sendo $L C_{2} \mathrm{o}$ limite de controle do segundo estágio.

A região dada por $\left(L A, L C_{1}\right)$ corresponde a região de advertência. A região acima de $L C_{1}$ é a região de ação do primeiro estágio e a região acima de $L C_{2}$ é a região de ação do segundo estágio.

Se um ponto cai na região de ação com o processo em controle, isto é, quando $\Sigma=\Sigma_{0}$, tem-se um alarme falso. Em outras palavras, o gráfico de controle sinaliza erroneamente a existência de uma causa especial. No primeiro estágio a probabilidade de se ter um alarme falso é dada por $\alpha_{1}$ e, no segundo estágio, por $\alpha_{2}$.

Se um ponto cai na região de ação após a ocorrência da causa especial, isto é, quando $\boldsymbol{\Sigma} \neq \boldsymbol{\Sigma}_{0}$, tem-se um alarme verdadeiro. No primeiro estágio a probabilidade de se ter um alarme verdadeiro é dada por $p_{1}$ e, no segundo estágio, por $p_{2}$.
Deste modo, $\alpha=\alpha_{1}+\alpha_{2}$ é a probabilidade de se ter um alarme falso quando a amostragem dupla está em uso e $p=p_{1}+p_{2}$ é o poder de detecção do gráfico de VMAX.

\section{Dara o caso l, o gráfico de VMAX tem um desempenho bem superior ao do gráfico de controle proposto por Alt (1985).}

Durante o período em controle, o número médio de itens inspecionado por amostragem, $\bar{n}$, é dado por

$$
\bar{n}=n_{1}+n_{2}\left(1-p_{0}\right)
$$

onde $p_{0}$ é a probabilidade de que a amostragem seja interrompida no primeiro estágio. Os valores de $\bar{n}, n_{1}$ e $n_{2}$ são especificados pelo usuário, onde $n_{1}<\bar{n}<n_{2}$. Por exemplo, se $\bar{n}=5, n_{1}=2$ e $n_{2}=10$, fazendo-se uso da expressão (5) tem-se que $p_{0}=0,7$, ou seja, com o processo em controle, $70 \%$ das vezes a amostragem é interrompida no primeiro estágio.

A Figura 2 mostra um gráfico de VMAX com os valores de $\operatorname{VMAX}_{1}$ e $\operatorname{VMAX}_{2}$ plotados. O gráfico de controle dá um sinal quando $\operatorname{VMAX}_{1}>L C_{1}$ ou quando $\operatorname{VMAX}_{2}>L C_{2}$. Os

Tabela 2: Valores de NMA para os gráficos de VMAX e de $|\mathrm{S}|$.

\begin{tabular}{|c|c|c|c|c|c|c|c|}
\hline \multirow[b]{5}{*}{$c^{2}$} & \multirow[b]{5}{*}{$L C$} & \multicolumn{6}{|c|}{$n$} \\
\hline & & \multicolumn{3}{|c|}{4} & \multicolumn{3}{|c|}{5} \\
\hline & & \multirow[t]{2}{*}{$|\mathbf{S}|$} & \multicolumn{2}{|c|}{ VMAX } & \multirow[t]{2}{*}{$|\mathbf{S}|$} & \multicolumn{2}{|c|}{ VMAX } \\
\hline & & & caso I & caso II & & caso I & caso II \\
\hline & & 6,134 & 4,094 & 4,094 & 5,375 & 3,668 & 3,668 \\
\hline 1,0 & & 200,0 & 200,0 & 200,0 & 200,0 & 200,0 & 200,0 \\
\hline 1,1 & & 146,8 & 136,6 & 143,0 & 141,4 & 132,5 & 139,7 \\
\hline 1,2 & & 112,5 & 92,4 & 107,0 & 104,6 & 86,8 & 102,4 \\
\hline 1,3 & & 89,1 & 63,9 & 82,9 & 80,5 & 58,3 & 78,0 \\
\hline 1,4 & & 73,3 & 45,7 & 66,1 & 64,1 & 40,7 & 61,4 \\
\hline 1,5 & & 60,4 & 33,9 & 54,1 & 51,9 & 29,6 & 49,6 \\
\hline 2,0 & & 30,2 & 11,6 & 25,4 & 24,1 & 9,62 & 22,3 \\
\hline 3,0 & & 13,6 & 4,09 & 10,7 & 10,2 & 3,38 & 9,09 \\
\hline 5,0 & & 6,37 & 1,95 & 4,77 & 4,58 & 1,67 & 3,98 \\
\hline
\end{tabular}


pontos amostrais $\operatorname{VMAX}_{1}$, ao caírem na região de advertência, disparam a inspeção de toda a amostra, e, nestes casos, a estatística $\operatorname{VMAX}_{2}$ é calculada e comparada com $L C_{2}$. Para evitar o uso de dois gráficos, um para o primeiro estágio e outro para o segundo estágio, o usuário pode construir o gráfico de VMAX com duas escalas, uma do lado esquerdo e uma do lado direito, como ilustrado na Figura 2. Os valores VMAX $_{1}$ correspondem aos pontos pretos que são plotados considerando a escala da esquerda e os valores $\operatorname{VMAX}_{2}$ correspondem aos pontos brancos que são plotados considerando a escala da direita.

\section{PROPRIEDADES DO GRÁFICO DE VMAX COM AMOSTRAGEM DUPLA}

Com a amostragem dupla, a eficiência do gráfico de controle em detectar uma alteração no processo é medida também pelo $N M A$, número médio de amostras até o sinal. A expressão do NMA para o gráfico de VMAX com amostragem dupla encontra-se no Apêndice II.

As tabelas desta seção foram obtidas fixando $L C_{1}=\infty$, ou seja, $\alpha_{1}=0$. Neste caso, o gráfico de controle dá um sinal so- mente quando $\operatorname{VMAX}_{2}>L C_{2}$. Os pontos amostrais $\operatorname{VMAX}_{1}$, ao caírem na região de advertência, que para o caso em que $L C_{1}=\infty$ corresponde à região acima de $L A$, disparam a inspeção de toda a amostra, e, nestes casos, a estatística $\operatorname{VMAX}_{2}$ é calculada e comparada com $L C_{2}$. Em geral, o desempenho do gráfico de VMAX tende a piorar sempre que se adotam valores de $\alpha_{1}>0$, ver Tabela 3 .

A Tabela 4 compara os gráficos de VMAX e de $|\mathbf{S}|$ com amostragem dupla. Os valores da Tabela 4 para o gráfico de $|\mathbf{S}|$ com amostragem dupla foram obtidos por Grigoryan e He (2005) por meio de simulações, ver Apêndice III. De acordo com a Tabela 4, observa-se que quando a amostragem dupla está em uso o gráfico proposto tem, em geral, um desempenho superior ao do gráfico de $|\mathbf{S}|$.

As Tabelas de 5 a 7 contêm os valores do NMA para o gráfico de VMAX com amostragem dupla. Nestas tabelas $n_{1}$ é fixo enquanto $n_{2}$ assume diferentes valores. Quando $n_{2}$ aumenta, o gráfico de VMAX se torna mais ágil na detecção de pequenas alterações; porém, se torna lento na detecção de grandes alterações. Por exemplo, da Tabela 5 observa-se que para $c^{2}=$ 1,3 o NMA diminui de 46,1 para 37,4 quando $n_{2}$ aumenta de 8 para 16 (caso I). Por outro lado, para $c^{2}=5$, o NMA aumenta de 1,53 para 1,67 quando $n_{2}$ passa de 8 para 16 (caso I).

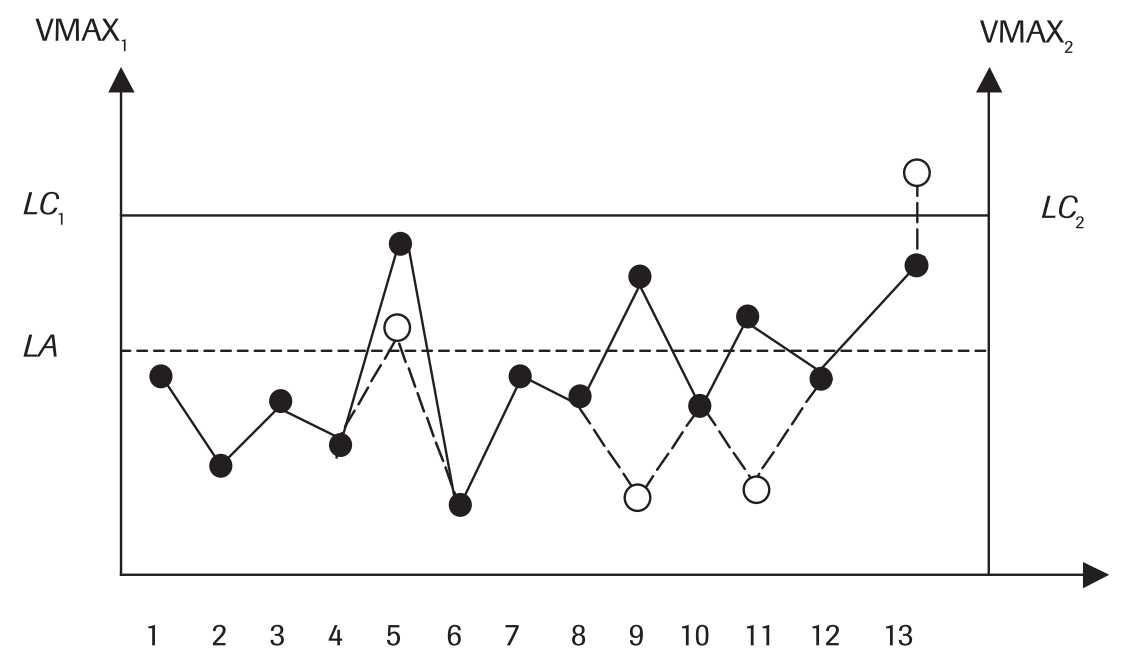

Número da amostra (I)

Valores de VMAX $_{1}$

Valores de VMAX $_{2}$

Figura 2: Gráfico de controle de VMAX com amostragem dupla. 
O efeito da escolha de $n_{1}$ na velocidade com que o gráfico de VMAX sinaliza o desajuste pode ser observado comparando os NMAs das Tabelas 6 e 7. Quando $n_{2}$ é pequeno e $n_{1}$ aumenta de 3 para 4 o gráfico de VMAX se mostra mais ágil. Por exemplo, observa-se que para $c^{2}=1,1$ e $n_{2}=10$, o NMA diminui de 65,6 para 65,0 quando $n_{1}$ passa de 3 para 4 (caso I). Para a mesma perturbação, porém para $n_{2}$ grande $\left(n_{2}=20\right)$, o $N M A$ aumenta de 73,8 para 75,3 quando $n_{1}$ passa de 3 para 4 . As Tabelas de 5 a 7 apresentam também os NMAs para o gráfico de $|\mathbf{S}|$ com amostragem dupla. Quando a amostragem dupla está em uso e apenas umas das variáveis é afetada pela causa especial (caso I), o gráfico de VMAX é sempre mais eficiente na detecção da perturbação do que o gráfico de $|\mathbf{S}|$. Porém, se as duas variáveis são afetadas pela causa especial (caso II) e o valor de $n_{2}$ é grande, o gráfico de VMAX tem desempenho inferior ao do gráfico de $|\mathbf{S}|$, exceto para grandes perturbações. Vale salientar que nesses casos, os desempenhos dos dois gráficos não são substancialmente diferentes e que o gráfico de VMAX é bem mais simples de ser utilizado, pois o usuário está muito mais familiarizado com o cálculo das variâncias amostrais do que com o do determinante de uma matriz de covariâncias.

Tabela 3: Valores de NMA para o gráfico de $\operatorname{VMAX}\left(\bar{n}=4, n_{1}=2, n_{2}=8, \rho=0,5\right)$.

\begin{tabular}{|c|c|c|c|c|c|c|c|}
\hline \multirow[b]{5}{*}{$c^{2}$} & & \multicolumn{2}{|c|}{$\alpha_{1}=0$} & \multicolumn{2}{|c|}{$\alpha_{1}=0,001$} & \multicolumn{2}{|c|}{$\alpha_{1}=0,0025$} \\
\hline & & caso I & caso II & caso I & caso II & caso I & caso II \\
\hline & $L A$ & 1,928 & 1,928 & 1,923 & 1,923 & 1,917 & 1,917 \\
\hline & $L C_{1}$ & $\infty$ & $\infty$ & 7,593 & 7,593 & 6,672 & 6,672 \\
\hline & $L C_{2}$ & 2,571 & 2,571 & 2,590 & 2,590 & 2,636 & 2,636 \\
\hline 1,0 & & 200,0 & 200,0 & 200,0 & 200,0 & 200,0 & 200,0 \\
\hline 1,1 & & 121,6 & 130,2 & 125,5 & 133,4 & 132,8 & 139,8 \\
\hline 1,2 & & 73,3 & 90,1 & 76,5 & 93,4 & 83,2 & 100,4 \\
\hline 1,3 & & 46,1 & 65,5 & 48,1 & 68,2 & 52,7 & 74,3 \\
\hline 1,4 & & 30,6 & 49,5 & 31,8 & 51,7 & 34,8 & 56,7 \\
\hline 1,5 & & 21,5 & 38,7 & 22,3 & 40,4 & 24,2 & 44,4 \\
\hline 2 & & 6,68 & 15,7 & 6,88 & 16,3 & 7,17 & 17,7 \\
\hline 3 & & 2,58 & 6,01 & 2,61 & 6,16 & 2,65 & 6,54 \\
\hline 5 & & 1,53 & 2,70 & 1,53 & 2,74 & 1,54 & 2,83 \\
\hline
\end{tabular}

Tabela 4: Valores de NMA para os gráficos de VMAX e de $|S|$ com amostragem dupla $(\bar{n}=4, \rho=0,5)$.

\begin{tabular}{|c|c|c|c|c|c|c|c|}
\hline \multirow[b]{2}{*}{$c^{2}$} & \multirow[b]{2}{*}{$n_{1}$} & \multirow[b]{2}{*}{$n_{2}$} & \multirow[b]{2}{*}{$L A$} & \multirow[b]{2}{*}{$L C_{2}$} & \multicolumn{2}{|c|}{ VMAX } & \multirow[t]{2}{*}{$|\mathbf{S}|$} \\
\hline & & & & & caso I & caso II & \\
\hline 1,1 & 3 & 23 & 3,175 & 1,701 & 108,6 & 118,9 & 102,0 \\
\hline 1,2 & 3 & 23 & 3,175 & 1,701 & 59,7 & 76,9 & 72,6 \\
\hline 1,3 & 3 & 23 & 3,175 & 1,701 & 35,6 & 53,2 & 49,5 \\
\hline 1,5 & 3 & 24 & 3,207 & 1,678 & 16,2 & 29,3 & 29,4 \\
\hline 2 & 3 & 19 & 3,031 & 1,818 & 5,62 & 11,9 & 14,0 \\
\hline 3 & 3 & 19 & 3,031 & 1,818 & 2,59 & 4,85 & 6,45 \\
\hline 5 & 3 & 11 & 2,613 & 2,193 & 1,50 & 2,47 & 3,59 \\
\hline
\end{tabular}


Machado, M. A. G.; de Magalhães, M. S.; Costa, A. F. B. Gráfico de controle de VMAX para o monitoramento da matriz de covariâncias. Produção, v. 18, n. 2, p. 222-239, 2008.

Tabela 5: Valores de NMA para os gráficos de VMAX e de $|S|\left(\bar{n}=4, n_{1}=3, \rho=0,5\right)$.

\begin{tabular}{|c|c|c|c|c|c|c|c|c|c|c|}
\hline \multirow[b]{6}{*}{$c^{2}$} & & \multicolumn{9}{|c|}{$n_{2}$} \\
\hline & & \multicolumn{3}{|c|}{8} & \multicolumn{3}{|c|}{12} & \multicolumn{3}{|c|}{16} \\
\hline & & \multirow[t]{2}{*}{$|\mathbf{S}|$} & \multicolumn{2}{|c|}{ VMAX } & \multirow[t]{2}{*}{$|\mathbf{S}|$} & \multicolumn{2}{|c|}{ VMAX } & \multirow[t]{2}{*}{$|\mathbf{S}|$} & \multicolumn{2}{|c|}{ VMAX } \\
\hline & & & caso I & caso II & & caso I & caso II & & caso I & caso II \\
\hline & $L A$ & 1,076 & 2,363 & 2,363 & 1,544 & 2,680 & 2,680 & 1,925 & 2,901 & 2,901 \\
\hline & $L C_{2}$ & 2,489 & 2,450 & 2,450 & 2,207 & 2,127 & 2,127 & 2,039 & 1,923 & 1,923 \\
\hline 1,0 & & 200,0 & 200,0 & 200,0 & 200,0 & 200,0 & 200,0 & 200,0 & 200,0 & 200,0 \\
\hline 1,1 & & 131,1 & 120,3 & 129,1 & 126,5 & 115,7 & 125,2 & 119,7 & 112,5 & 122,3 \\
\hline 1,2 & & 93,8 & 71,7 & 88,8 & 86,0 & 66,7 & 84,0 & 79,1 & 63,3 & 80,7 \\
\hline 1,3 & & 70,3 & 44,7 & 64,2 & 62,3 & 40,6 & 59,6 & 55,9 & 38,0 & 56,5 \\
\hline 1,4 & & 55,2 & 29,5 & 48,3 & 46,3 & 26,4 & 44,2 & 41,7 & 24,7 & 41,6 \\
\hline 1,5 & & 43,9 & 20,6 & 37,6 & 37,3 & 18,4 & 34,0 & 32,2 & 17,2 & 31,8 \\
\hline 2 & & 19,8 & 6,38 & 15,1 & 16,0 & 5,81 & 13,3 & 13,7 & 5,62 & 12,4 \\
\hline 3 & & 9,09 & 2,48 & 5,76 & 7,19 & 2,43 & 5,13 & 6,39 & 2,50 & 4,89 \\
\hline 5 & & 4,68 & 1,47 & 2,60 & 4,04 & 1,52 & 2,46 & 3,64 & 1,58 & 2,48 \\
\hline
\end{tabular}

Tabela 6: Valores de NMA para os gráficos de VMAX e de $|S|\left(\bar{n}=5, n_{1}=3, \rho=0,5\right)$.

\begin{tabular}{|c|c|c|c|c|c|c|c|c|c|c|}
\hline \multirow[b]{6}{*}{$c^{2}$} & \multirow[b]{5}{*}{$L A$} & \multicolumn{9}{|c|}{$n_{2}$} \\
\hline & & \multicolumn{3}{|c|}{10} & \multicolumn{3}{|c|}{15} & \multicolumn{3}{|c|}{20} \\
\hline & & \multirow[t]{2}{*}{$|\mathbf{S}|$} & \multicolumn{2}{|c|}{ VMAX } & \multirow[t]{2}{*}{$|\mathbf{S}|$} & \multicolumn{2}{|c|}{ VMAX } & \multirow[t]{2}{*}{$|\mathbf{S}|$} & \multicolumn{2}{|c|}{ VMAX } \\
\hline & & & caso I & caso II & & caso I & caso II & & caso I & caso II \\
\hline & & 0,644 & 1,984 & 1,984 & 1,025 & 2,312 & 2,312 & 1,328 & 2,538 & 2,538 \\
\hline & $L C_{2}$ & 2,326 & 2,334 & 2,334 & 2,064 & 2,038 & 2,038 & 1,922 & 1,855 & 1,855 \\
\hline 1,0 & & 200,0 & 200,0 & 200,0 & 200,0 & 200,0 & 200,0 & 200,0 & 200,0 & 200,0 \\
\hline 1,1 & & 128,1 & 115,1 & 124,9 & 120,0 & 109,3 & 119,9 & 114,1 & 105,2 & 116,4 \\
\hline 1,2 & & 89,4 & 65,6 & 83,6 & 79,6 & 59,6 & 77,8 & 75,6 & 55,6 & 73,8 \\
\hline 1,3 & & 65,2 & 39,4 & 59,1 & 56,6 & 34,7 & 53,7 & 51,2 & 31,8 & 50,1 \\
\hline 1,4 & & 50,0 & 25,3 & 43,6 & 42,3 & 21,9 & 38,9 & 37,4 & 20,0 & 35,9 \\
\hline 1,5 & & 39,9 & 17,4 & 33,4 & 32,5 & 14,9 & 29,4 & 28,4 & 13,6 & 26,9 \\
\hline 2 & & 18,0 & 5,21 & 12,8 & 14,0 & 4,63 & 10,9 & 12,1 & 4,43 & 9,88 \\
\hline 3 & & 7,87 & 2,10 & 4,76 & 6,48 & 2,07 & 4,12 & 5,71 & 2,14 & 3,87 \\
\hline 5 & & 4,23 & 1,34 & 2,19 & 3,73 & 1,40 & 2,05 & 3,51 & 1,46 & 2,07 \\
\hline
\end{tabular}




\section{EXEMPLO ILUSTRATIVO}

Nesta seção, considera-se o exemplo citado por Aparisi et al. (2001) para explicar o uso do gráfico de controle com amostragem dupla baseado na estatística VMAX ou na estatística $|\mathbf{S}|$.

A peça da Figura 3 tem duas dimensões a serem controladas, $X$ (distância entre centros de dois furos interiores) e $Y$ (distância entre dois centros de furos laterais). As duas características de qualidade são correlacionadas, sendo $\rho=0,7$

Quando o processo está em controle, o vetor de médias e a matriz de covariâncias do processo são conhecidos:

$$
\boldsymbol{\mu}_{0}=\left(\begin{array}{l}
10,0 \\
10,5
\end{array}\right), \boldsymbol{\Sigma}_{0}=\left(\begin{array}{cc}
0,45 & 0,332 \\
0,332 & 0,45
\end{array}\right) .
$$

Inicialmente foram geradas 10 amostras com o processo em controle, onde $\bar{n}=4, n_{1}=3$ e $n_{2}=8$. A última amostra foi gerada sob a condição do caso I ter ocorrido, em que a causa especial aumenta somente a variabilidade da primeira variável $X$, sendo $c^{2}=3,0$. Portanto, $\boldsymbol{\Sigma}_{1}=\left(\begin{array}{cc}1,35 & 0,575 \\ 0,575 & 0,45\end{array}\right)$.

\section{Neste artigo, considerou-se um gráfico de controle baseado nas variâncias amostrais} de duas características de qualidade.

Na Tabela 8 estão os dados de $\left(X_{i}, Y_{i}\right)$ e na Tabela 9 estão as variâncias amostrais $S_{1 x}^{2}, S_{1 y}^{2}, S_{2 x}^{2}$ e $S_{2 y}^{2}$ e as estatísticas VMAX $\operatorname{VMAX}_{2},\left|\mathbf{S}_{1}\right|$ e $\left|\mathbf{S}_{2}\right|$. É importante salientar que $\left|\mathbf{S}_{1}\right|$ é o deter-

Tabela 7: Valores de NMA para os gráficos de VMAX e de $|S|\left(\bar{n}=5, n_{1}=4, \rho=0,5\right)$.

\begin{tabular}{|c|c|c|c|c|c|c|c|c|c|c|}
\hline \multirow[b]{6}{*}{$c^{2}$} & \multirow[b]{5}{*}{$L A$} & \multicolumn{9}{|c|}{$n_{2}$} \\
\hline & & \multicolumn{3}{|c|}{10} & \multicolumn{3}{|c|}{15} & \multicolumn{3}{|c|}{20} \\
\hline & & \multirow[t]{2}{*}{$|\mathbf{S}|$} & \multicolumn{2}{|c|}{ VMAX } & \multirow[t]{2}{*}{$|\mathbf{S}|$} & \multicolumn{2}{|c|}{ VMAX } & \multirow[t]{2}{*}{$|\mathbf{S}|$} & \multicolumn{2}{|c|}{ VMAX } \\
\hline & & & caso I & caso II & & caso I & caso II & & caso I & caso II \\
\hline & & 1,694 & 2,319 & 2,319 & 2,151 & 2,574 & 2,574 & 2,507 & 2,750 & 2,750 \\
\hline & $L C_{2}$ & 2,487 & 2,240 & 2,240 & 2,193 & 1,963 & 1,963 & 2,028 & 1,787 & 1,787 \\
\hline 1,0 & & 200,0 & 200,0 & 200,0 & 200,0 & 200,0 & 200,0 & 200,0 & 200,0 & 200,0 \\
\hline 1,1 & & 127,3 & 114,5 & 124,4 & 116,3 & 109,9 & 120,4 & 111,6 & 106,8 & 117,6 \\
\hline 1,2 & & 85,9 & 65,0 & 83,0 & 73,6 & 60,3 & 78,4 & 66,7 & 57,4 & 75,3 \\
\hline 1,3 & & 60,3 & 39,0 & 58,5 & 51,0 & 35,4 & 54,3 & 44,5 & 33,4 & 51,5 \\
\hline 1,4 & & 44,3 & 25,0 & 43,2 & 36,4 & 22,5 & 39,5 & 31,6 & 21,2 & 37,2 \\
\hline 1,5 & & 34,5 & 17,2 & 33,1 & 27,6 & 15,4 & 29,9 & 23,8 & 14,6 & 28,1 \\
\hline 2 & & 13,9 & 5,17 & 12,7 & 10,9 & 4,83 & 11,2 & 8,98 & 4,79 & 10,6 \\
\hline 3 & & 5,66 & 2,09 & 4,71 & 4,54 & 2,12 & 4,30 & 3,95 & 2,21 & 4,21 \\
\hline 5 & & 2,92 & 1,32 & 2,19 & 2,54 & 1,38 & 2,14 & 2,35 & 1,42 & 2,21 \\
\hline
\end{tabular}

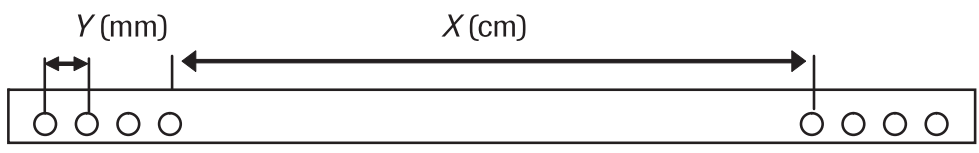

Figura 3: Peça do exemplo ilustrativo. 
minante de $\mathbf{S}$ considerando uma amostra de tamanho $n_{1}$ e $\left|\mathbf{S}_{2}\right|$ de tamanho $n$, e que os valores plotados no gráfico de $|\mathbf{S}|$ são os das estatísticas $\left|\mathbf{S}_{1}\right|$ e $\left|\mathbf{S}_{2}\right|$ e não seus valores padronizados. Para a obtenção do limite de controle $L C_{2}=1,103$ e do limite de advertência $L A=1,063$ do gráfico de VMAX foi utilizada a expressão (5) e as expressões (A2) e (A5) do Apêndice II, para $a=b=1,0$. O limite de controle $L C_{2}=0,230$ e o limite de advertência $L A=0,099$ do gráfico de $|\mathbf{S}|$ foram obtidos por simulação, de acordo com o esquema de amostragem dupla. Neste exemplo, adotou-se $\alpha=0,005$ e $L C_{1}=\infty$.

As Figuras 4 e 5 apresentam os gráficos de VMAX e de $|\mathbf{S}|$ com amostragem dupla. O gráfico de VMAX sinaliza já na $1^{a}$ amostra após a ocorrência da causa especial. Entretanto, o mesmo não ocorre com o gráfico de $|\mathbf{S}|$.

\section{CONCLUSÕES}

Neste artigo, considerou-se um gráfico de controle baseado nas variâncias amostrais de duas características de qualidade. Os pontos plotados no gráfico correspondem ao valor da maior variância. A principal razão em considerar

Tabela 8: Dados para o exemplo ilustrativo.

\begin{tabular}{|c|c|c|c|c|c|c|c|c|c|c|c|c|}
\hline$\#$ & & 1 & 2 & 3 & 4 & 5 & 6 & 7 & 8 & 9 & 10 & 11 \\
\hline \multirow[t]{2}{*}{1} & $X(\mathrm{~cm})$ & 11,009 & 10,307 & 9,027 & - & - & - & - & - & - & - & - \\
\hline & $\mathrm{Y}(\mathrm{mm})$ & 10,844 & 10,416 & 10,093 & - & - & - & - & - & - & - & - \\
\hline \multirow[t]{2}{*}{2} & & 9,546 & 9,362 & 9,857 & - & - & - & - & - & - & - & - \\
\hline & & 11,030 & 9,678 & 10,577 & - & - & - & - & - & - & - & - \\
\hline \multirow[t]{2}{*}{3} & & 10,850 & 10,337 & 10,398 & - & - & - & - & - & - & - & - \\
\hline & & 10,440 & 10,266 & 11,172 & - & - & - & - & - & - & - & - \\
\hline \multirow[t]{2}{*}{4} & & 10,904 & 10,003 & 10,502 & - & - & - & - & - & - & - & - \\
\hline & & 10,786 & 11,028 & 11,188 & - & - & - & - & - & - & - & - \\
\hline \multirow[t]{2}{*}{5} & & 9,501 & 10,345 & 9,571 & 9,591 & 10,33 & 10,55 & 10,751 & 10,9 & 8,979 & 10,47 & 10,705 \\
\hline & & 10,584 & 11,389 & 12,08 & 10,61 & 10,24 & 10,54 & 10,585 & 11,59 & 9,803 & 11,43 & 11,324 \\
\hline \multirow[t]{2}{*}{6} & & 10,840 & 10,543 & 10,670 & - & - & - & - & - & - & - & - \\
\hline & & 10,181 & 10,917 & 11,022 & - & - & - & - & - & - & - & - \\
\hline \multirow[t]{2}{*}{7} & & 9,909 & 10,270 & 9,395 & - & - & - & - & - & - & - & - \\
\hline & & 9,894 & 10,627 & 10,111 & - & - & - & - & - & - & - & - \\
\hline \multirow[t]{2}{*}{8} & & 10,509 & 10,815 & 11,227 & - & - & - & - & - & - & - & - \\
\hline & & 9,960 & 10,405 & 11,405 & - & - & - & - & - & - & - & - \\
\hline \multirow[t]{2}{*}{9} & & 9,943 & 8,816 & 10,204 & - & - & - & - & - & - & - & - \\
\hline & & 10,062 & 9,627 & 9,891 & - & - & - & - & - & - & - & - \\
\hline \multirow[t]{2}{*}{10} & & 8,920 & 9,672 & 8,865 & - & - & - & - & - & - & - & - \\
\hline & & 9,544 & 10,708 & 10,191 & - & - & - & - & - & - & - & - \\
\hline \multirow[t]{2}{*}{11} & & 10,889 & 11,43 & 11,07 & 9,613 & 8,441 & 7,761 & 9,673 & 11,62 & 11,19 & 9,954 & 12,372 \\
\hline & & 10,763 & 9,35 & 9,273 & 10,84 & 9,196 & 9,209 & 10,607 & 11,65 & 10,74 & 10,51 & 11,576 \\
\hline
\end{tabular}


o gráfico proposto é que ele possui uma melhor capacidade de diagnóstico, ou seja, com ele é mais fácil identificar a variável que teve sua variabilidade alterada pela ocorrência da causa especial.

O gráfico proposto é sempre mais eficiente que o gráfico da variância amostral generalizada $|\mathbf{S}|$. Quando a amostragem dupla está em uso, observa-se que o gráfico proposto também é mais rápido do que o gráfico de $|\mathbf{S}|$, exceto em alguns casos em que o tamanho da amostra no segundo estágio é muito grande.

Tabela 9: Valores de $S_{1 x}^{2}, S_{1 y}^{2}, S_{2 x}^{2}, S_{2 y}^{2}, \operatorname{VMAX}_{1}, \operatorname{VMAX}_{2},\left|\mathrm{~S}_{1}\right| \mathrm{e}\left|\mathrm{S}_{2}\right|$.

\begin{tabular}{|c|c|c|c|c|c|c|c|c|}
\hline \multirow[b]{2}{*}{ \# } & \multicolumn{4}{|c|}{ 1ํestágio } & \multicolumn{4}{|c|}{ 2o estágio } \\
\hline & $S_{1 x}^{2}$ & $S_{1 y}^{2}$ & VMAX $_{1}$ & $\left|\mathbf{S}_{1}\right|$ & $S_{2 x}^{2}$ & $S_{2 y}^{2}$ & $\operatorname{VMAX}_{2}$ & $\left|\mathbf{S}_{2}\right|$ \\
\hline 1 & 0,686 & 0,097 & 0,686 & 0,083 & - & - & - & - \\
\hline 2 & 0,211 & 0,321 & 0,321 & 0,026 & - & - & - & - \\
\hline 3 & 0,331 & 0,170 & 0,331 & 0,018 & - & - & - & - \\
\hline 4 & 0,356 & 0,278 & 0,356 & 0,007 & - & - & - & - \\
\hline 5 & 0,184 & 1,096 & 1,096 & 0,122 & 0,387 & 0,600 & 0,600 & 0,165 \\
\hline 6 & 0,483 & 0,183 & 0,483 & 0,003 & - & - & - & - \\
\hline 7 & 0,149 & 0,178 & 0,178 & 0,023 & - & - & - & - \\
\hline 8 & 0,810 & 0,373 & 0,810 & 0,040 & - & - & - & - \\
\hline 9 & 0,482 & 0,442 & 0,482 & 0,018 & - & - & - & - \\
\hline 10 & 0,854 & 0,351 & 0,854 & 0,050 & - & - & - & - \\
\hline 11 & 1,327 & 0,966 & 1,327 & 0,041 & 1,941 & 0,813 & 1,941 & - \\
\hline
\end{tabular}

\#: Número da amostra

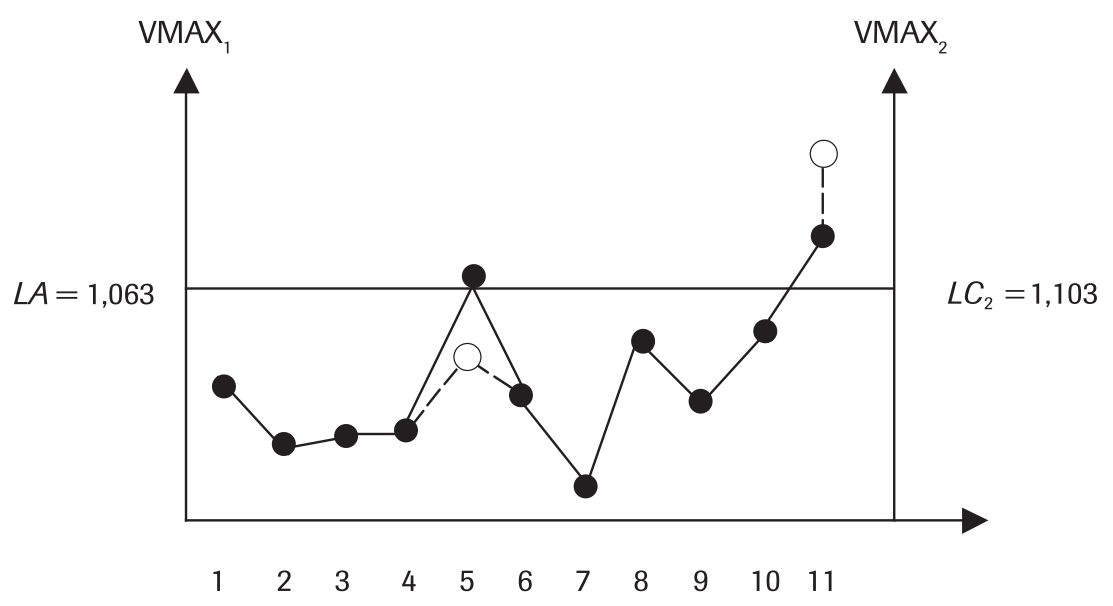

Número da amostra (I) 
Com a crescente informatização dos controles "online", tornou-se usual acompanhar as condições do processo por meio do monitoramento simultâneo de muitos parâmetros de qualidade. Sendo assim, a extensão natural do presente trabalho é a criação de um gráfico de VMAX para o monitoramento de processos com $p$ variáveis de qualidade, sendo $p>2$. Os autores já estão trabalhando nesta direção. Uma alternativa para o controle de processos multivariados consiste em se reduzir o número de variáveis a serem monitoradas, através, por exemplo, da aplicação da técnica de análise de componentes principais (SOUZA; RIGÃO, 2005).

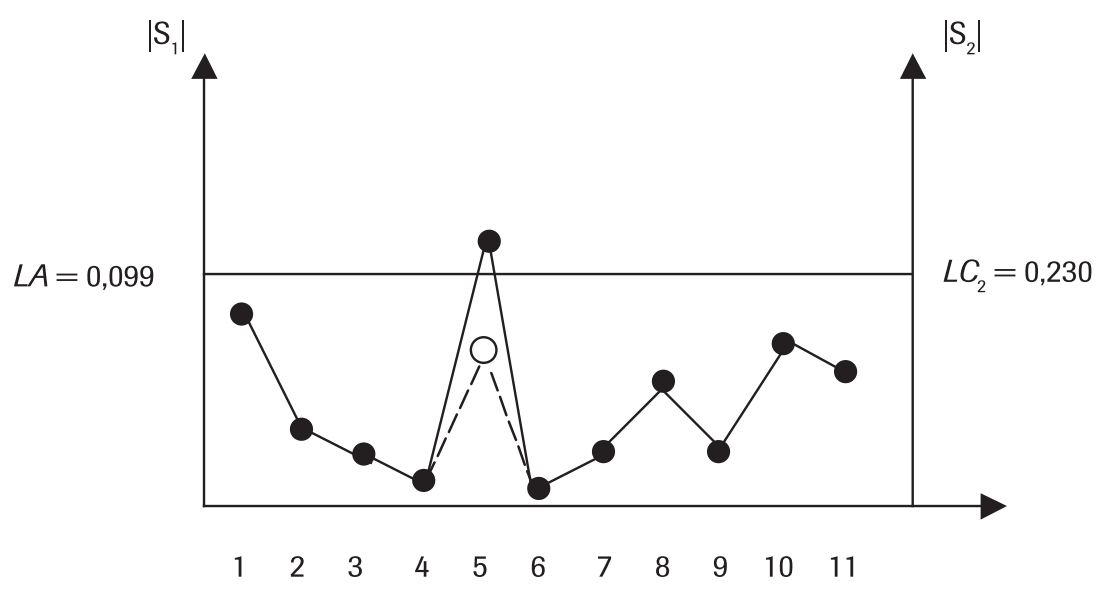

Número da amostra (i)

- Valores de $\left|\mathrm{S}_{1}\right| \quad O$ Valores de $\left|\mathrm{S}_{2}\right|$

Figura 5: Gráfico de $|\mathbf{S}|$ com amostragem dupla - Exemplo. 


\section{REFERÊNCIAS}

ALT, F. B. Multivariate quality control. In: Kotz, S., Johnson, N. L., ed., Encyclopedia of Statistical Sciences. Wiley. 1985.

ANDERSON, T. W. An Introduction to Multivariate Statistical Analysis. Stanford: John Wiley. 2003.

APARISI, F.; JABALOYES, J.; CARRIÓN, A. Statistical properties of the $|\mathrm{S}|$ multivariate control chart. Communications in Statistics - Theory and Methods, v. 28, p. 2671-2686, 1999.

APARISI, F.; JABALOYES, J.; CARRIÓN, A. Generalized variance chart design with adaptive sample sizes. The bivariate case. Communications in Statistics - Simulation and Computation, v. 30, p. 931-948, 2001.

CAROT, V.; JABALOYES, J.M.; CAROT, T. Combined double sampling and variable sampling interval $\bar{X}$ chart. International Journal of Production Research, v. 40, p. 2175-2186, 2002.

CHANG, S. I.; ZHANG, K. Statistical process control for variance shift detections of multivariate autocorrelated processes. Quality Technology of Quantitative Management, v. 4, p. 413-435, 2007.

CHEN, G.; CHENG, S. W.; XIE, H. Monitoring process mean and variability with one EWMA chart. Journal of Quality Technology, v. 33, p. 223-233, 2001.

COSTA, A. F. B. $\bar{X}$ charts with variable sample size. Journal of Quality Technology, v. 26, p. 155-163, 1994.

COSTA, A. F. B. Joint $\bar{X}$ and $\mathrm{R}$ charts with variable sample sizes and sampling intervals. Journal of Quality Technology, v. 31, p. 387-397, 1999.

COSTA, A. F. B.; MACHADO, M. A. G. Synthetic control chart with two-stage sampling for monitoring bivariate processes. Pesquisa Operacional, v. 27 , p. 117-130, 2007.

COSTA, A. F. B.; RAHIM, M. A. A single EWMA chart for monitoring process mean and process variance. Quality Technology \& Quantitative Management, v. 3, p. 295-305, 2006.
COSTA, A.F.B.; EPPRECHT E.K.; CARPINETTI, L.C.R. Controle Estatístico de Qualidade. 2. ed. São Paulo: Editora Atlas, 2005. 334 p.

COSTA, A.F.B.; De MAGALHÃES, M.S.; EPPRECHT E.K. The non-central chi-square chart with double sampling. Brazilian Journal of Operation and Production Management, v. 2, p. $57-80,2005 a$.

CROASDALE, R. Control charts for a double sampling scheme based on average production run length. International Journal of Production Research, v. 12, p. 585-592, 1974.

DAUDIN, J. J. Double sampling $\bar{X}$ charts. Journal of Quality Technology, v. 24, p. 78-87, 1992.

De MAGALHÃES, M. S.; COSTA, A. F. B.; EPPRECHT, E. K. Constrained optimization model for the design of an adaptive $\bar{X}$ chart. International Journal of Production Research, v. 40, p. 3199-3218, 2002.

De MAGALHÃES, M. S.; COSTA, A. F. B.; MOURA NETO, F. D. Adaptive control charts: a Markovian approach for processes subject to independent out-of-control disturbances. International Journal of Production Economics, v. 99, p. 236-246, 2006.

DJAUHARI, M. A. Improved monitoring of multivariate process variability. Journal of Quality Technology, v. 37, p. 32-39, 2005.

DOMANGUE, R.; PATCH, SC. Some omnibus exponentially weighted moving average statistical process monitoring schemes. Technometrics, v. 33, p. 299-313, 1991.

GRIGORYAN, A.; HE, D. Multivariate double sampling $|\mathrm{S}|$ charts for controlling process variability. International Journal of Production Research, v. 43, p. 715-730, 2005.

HAWKINS, D. M.; OLWELL, D. H. Cumulative sum charts and charting for quality improvement. New York: Springer-Verlag. 1998.

HE, D; GRIGORYAN, A. Construction of double sampling S- control charts for agile manufacturing. Quality and Reliability Engineering International, v. 18, p. 343-355, 2002.
HE, D; GRIGORYAN, A. Multivariate multiple sampling charts. IIE Transactions, v. 37, p. 509521, 2005.

HE, D; GRIGORYAN, A. Joint statistical design of double sampling $\bar{X}$ and $\mathrm{S}$ charts. European Journal of Operational Research, v. 168, p. 122142, 2006.

HE, D; GRIGORYAN, A; SIGH, M. Design of double and triple sampling $\bar{X}$ control charts using genetic algorithms. International Journal of Production Research, v. 40, p. 1387-1404, 2002.

IRIANTO, D.; SHINOZAKI, N. An optimal double sampling $\bar{X}$ control chart. International Journal of Industrial Engineering - Applications and Practice, v. 5, p. 226-234, 1998.

LUCAS, J. M.; SACCUCCI, M. S. Exponentially weighted moving average control schemes: properties and enhancements. Technometrics, v. 32, p. 1-16, 1990.

MACHADO, M. A. G. Estudo das propriedades dos gráficos de controle bivariados com amostragem dupla. Dissertação (Mestrado em Engenharia Mecânica) - Faculdade de Engenharia do Campus de Guaratinguetá, Universidade Estadual Paulista, Guaratinguetá, $129 f ., 2006$.

MASON, R. L.; YOUNG, J. C. Multivariate Statistical Process Control with Industrial Applications. Philadelphia: ASA - SIAM. 2002.

MICROSOFT FORTRAN POWER STATION 4.0. Professional Edition with Microsoft IMSL Mathematical and Statistical Libraries, Microsoft Corporation, 1995.

MONTGOMERY, D. C. Introduction to Statistical Quality Control. New York: John Wiley \& Sons, Inc. 2004.

PAGE, E. S. Continuous inspection schemes. Biometrika, v. 41, p. 100-115, 1954.

REYNOLDS, M. R., Jr.; STOUMBOS, Z. G. Should exponentially weighted moving average and cumulative sum charts be used with Shewhart limits? Technometrics, v. 47, p. 409424, 2005. 
SEREL, D. A.; MOSKOWITZ, H.; TANG, J. Univariate $\bar{X}$ control charts for individual characteristics in a multinormal model. IIE Transactions, v. 32, p. 1115-1125, 2000.

SOUZA, A. M.; RIGÃO, M. H. Identificação de variáveis fora de controle em processos produtivos multivariados. Revista Produção, v. 15, p. 74-86, 2005.

SURTIHADI, J.; RAGHAVACHARI, M.; RUNGER, G. Multivariate control charts for process dis- persion. International Journal of Production Research, v. 42, p. 2993-3009, 2004.

YEH, A. B.; LIN, D. K. J.; ZHOU, H.; VENKATARAMANI, C. A multivariate exponentially weighted moving average control chart for monitoring process variability. Journal of Applied Statistics, v. 30, p. 507-536, 2003

YEH, A. B.; HUWANG, L.; WU, Y. F. A likelihoodratio-based EWMA control chart for monitoring variability of multivariate normal processes. /l Transactions, v. 36, p. 865-879, 2004.

YEH, A. B.; HUWANG, L.; WU, C. W. A multivariate EWMA control chart for monitoring process variability with individual observations. II Transactions, v. 37, p. 1023-1035, 2005.

YEH, A. B.; LIN, D. K. J.; MCGRATH, R. N. Multivariate control charts for monitoring covariance matrix: a review. Quality Technology and Quantitative Management, v. 3, p. 415-436, 2006.

\section{AGRADECIMENTOS}

Os autores agradecem aos dois revisores anônimos pelos úteis comentários e sugestões. Esta pesquisa contou com o apoio da FAPESP e do CNPq.

\section{SOBRE OS AUTORES}

\section{Marcela Aparecida Guerreiro Machado}

Departamento de Produção/FEG/UNESP,

End.: Avenida Ariberto Pereira da Cunha, 333 - CEP 12516-410 - Bairro Pedregulho - Guaratinguetá - SP.

E-mail:marcela@feg.unesp.br

\section{Maysa Sacramento de Magalhães}

ENCE/IBGE

End.: Rua André Cavalcanti, 106 - CEP 20231-050 - Santa Teresa - Rio de Janeiro - RJ.

E-mail: maysa.magalhaes@ibge.gov.br

\section{Antônio Fernando Branco Costa}

Departamento de Produção/FEG/UNESP,

End.: Avenida Ariberto Pereira da Cunha, 333 - CEP 12516-410 - Bairro Pedregulho - Guaratinguetá - SP.

E-mail: fbranco@feg.unesp.br 


\section{APÊNDICE I: OBTENÇÃO DO NMA PARA O GRÁFICO DE VMAX}

Considere as estatísticas $S_{x}^{2}=\frac{\sum_{i=1}^{n} x_{i}{ }^{2}}{n}$ e $S_{y}^{2}=\frac{\sum_{i=1}^{n} y_{i}{ }^{2}}{n}$, onde $x_{i}=X_{i}-\mu_{x}, y_{i}=Y_{i}-\mu_{y}$ e $X$ e $Y$ são duas características de qualidade que seguem uma distribuição normal bivariada. O poder do gráfico de VMAX é dado por:

$$
p=1-\operatorname{Pr}\left[\left(S_{x}^{2}<L C \sigma_{0 x}^{2}\right) \cap\left(S_{y}^{2}<L C \sigma_{0 y}^{2}\right)\right],
$$

onde LC é o fator de abertura dos limites de controle. Portanto,

$$
N M A=\frac{1}{1-\operatorname{Pr}\left[\left(S_{x}^{2}<L C \sigma_{0 x}^{2}\right) \cap\left(S_{y}^{2}<L C \sigma_{0 y}^{2}\right)\right]} .
$$

Sem perda de generalidade, supõe-se que com o processo em controle a matriz de covariâncias é dada por $\boldsymbol{\Sigma}_{0}=\left(\begin{array}{cc}1 & \sigma_{x y} \\ \sigma_{y x} & 1\end{array}\right)$. A correlação entre $X$ e $Y$, dada por $\rho=\frac{\sigma_{x y}}{\sigma_{x} \sigma_{y}}$, não é afetada pela causa especial. Da maneira como $\Sigma_{0}$ foi definida, tem-se que $\sigma_{0 x}=\sigma_{0 y}=1$ e, conseqüentemente, $\rho=\sigma_{x y}=\sigma_{y x}$.

A ocorrência da causa especial altera a matriz de covariâncias inicial para $\boldsymbol{\Sigma}_{1}=\left(\begin{array}{cc}a \cdot a & a \cdot b \cdot \sigma_{x y} \\ a \cdot b \cdot \sigma_{y x} & b \cdot b\end{array}\right)$, sendo. $\sigma_{x y}=\sigma_{y x}=\rho$. Para o caso I $a=c$ e $b=1$ (ou $b=c$ e $a=1$ ) e para o caso II $a=b=\sqrt{c}$. Se $X$ e $Y$ são normalmente distribuídos,

$$
\frac{n S_{x}^{2}}{a^{2}}=\sum_{i=1}^{n}\left(\frac{x_{i}}{a}\right)^{2} \sim \chi_{\mathrm{n}}^{2} \quad \text { e } \quad \frac{y_{\mathrm{i}} \mid x_{\mathrm{i}}}{b \sqrt{1-\rho^{2}}} \sim N\left(\frac{\rho x_{i}}{a \sqrt{1-\rho^{2}}}, 1\right) .
$$

Conseqüentemente,

e

$$
\frac{n S_{y}^{2} \mid x_{1}, x_{2}, \ldots, x_{n}}{b^{2}\left(1-\rho^{2}\right)}=\sum_{i=1}^{n}\left(\frac{y_{i} \mid x_{i}}{b \sqrt{1-\rho^{2}}}\right)^{2} \sim \chi_{n,\left(\rho^{2} / 1-\rho^{2}\right) \chi_{n}^{2}}^{2}
$$

$$
p=1-\int_{0}^{n L C / a^{2}} \operatorname{Pr}\left[\chi_{n,\left(t \rho^{2} / 1-\rho^{2}\right)}^{2}<\frac{n L C}{b^{2}\left(1-\rho^{2}\right)}\right] \frac{1}{2^{n / 2} \Gamma(n / 2)} e^{-t / 2} t^{(n / 2)-1} d t,
$$

lembrando que a notação $\chi_{n,\left(\rho^{2} / 1-\rho^{2}\right) x_{n}^{2}}^{2}$ representa uma distribuição de Qui-quadrado com $n$ graus de liberdade e com o parâmetro de não-centralidade dado por $\left(\rho^{2} / 1-\rho^{2}\right) \chi_{n}^{2}$.

O limite de controle, LC, é obtido pela expressão (A1), fazendo $a=b=1$ e $p=\alpha$. Utilizou-se a sub-rotina CSNDF da biblioteca IMSL FORTRAN (1995) para o cálculo da função de distribuição de qui-quadrado não-central. 


\section{APÊNDICE II: OBTENÇÃO DO NMA PARA O GRÁFICO DE VMAX COM AMOSTRAGEM DUPLA}

Sejam as variâncias amostrais $S_{1 x}^{2}=\frac{\sum_{i=1}^{n_{1}} x_{i}{ }^{2}}{n_{1}}, S_{1 y}^{2}=\frac{\sum_{i=1}^{n_{1}} y_{i}{ }^{2}}{n_{1}}, S_{2 x}^{2}=\frac{\sum_{i=1}^{n} x_{i}{ }^{2}}{n}$ e $S_{2 y}^{2}=\frac{\sum_{i=1}^{n} y_{i}{ }^{2}}{n}$ onde $x_{i}=X_{i}-\mu_{x}, y_{i}=Y_{i}-\mu_{y} \mathrm{e}$

$X$ e $Y$ são duas características de qualidade que seguem uma distribuição normal bivariada.

De acordo com o Apêndice I, a probabilidade $p_{1}$ do gráfico de controle sinalizar um desajuste do processo após a inspeção da amostra de tamanho $n_{1}$ é dada por:

$$
\begin{gathered}
p_{1}=1-\operatorname{Pr}\left[\left(S_{1 x}^{2}<L C_{1}\right) \cap\left(S_{1 y}^{2}<L C_{1}\right)\right], \\
p_{1}=1-\int_{0}^{n_{1} L C_{1} / a^{2}} \operatorname{Pr}\left[\chi_{n_{1}, t_{1}\left(\rho^{2} / 1-\rho^{2}\right)}^{2}<\frac{n_{1} L C_{1}}{b^{2}\left(1-\rho^{2}\right)}\right] f\left(t_{1}\right) d t_{1} .
\end{gathered}
$$

O limite de controle do primeiro estágio, $L C_{1}$, é obtido pela expressão (A2), fazendo $p_{1}=\alpha_{1}$ e $a=b=1$.

A probabilidade $p_{s}$, da amostragem ser interrompida no primeiro estágio, é dada por:

$$
p_{s}=p_{1}+\int_{0}^{n_{1} L A / a^{2}} \operatorname{Pr}\left[\chi_{n_{1}, t_{1}\left(\rho^{2} / 1-\rho^{2}\right)}^{2}<\frac{n_{1} L A}{b^{2}\left(1-\rho^{2}\right)}\right]\left(t_{1}\right) d t_{1}
$$

O limite de advertência, $L A$, é obtido pela expressão (A3), fazendo $p_{s}=p_{0}, p_{1}=\alpha_{1}$ e $a=b=1$. O valor de $\alpha_{1}$ é especificado pelo usuário e o valor de $p_{0}$ é função de $n_{1}, n_{2}$ e $\bar{n}$ (ver expressão (5)), que também devem ser fornecidos pelo usuário.

A probabilidade $p_{2}$, do gráfico de controle sinalizar um desajuste após a inspeção dos $n_{1}+n_{2}$ itens da amostra, é dada por:

$$
p_{2}=1-\left(p_{2 a}+p_{2 b}\right)
$$

onde

$$
\begin{gathered}
p_{2 a}=\operatorname{Pr}\left[\left(S_{2 x}^{2}<L C_{2}\right) \cap\left(S_{2 y}^{2}<L C_{2}\right) \mid\left(S_{1 x}^{2}<L A\right) \cap\left(L A<S_{1 y}^{2}<L C_{1}\right)\right] \cdot \operatorname{Pr}\left[\left(S_{1 x}^{2}<L A\right) \cap\left(L A<S_{1 y}^{2}<L C_{1}\right)\right] \\
=\int_{0}^{n_{1} L A / a^{2}} \int_{0}^{n L C_{2} / a^{2}-t_{1}} \operatorname{Pr}\left[\chi_{n,\left(t_{1}+t_{2}\right)\left(\rho^{2} / 1-\rho^{2}\right)}^{2}<\frac{n L C_{2}}{b^{2}\left(1-\rho^{2}\right)} \mid \frac{n_{1} L A}{b^{2}\left(1-\rho^{2}\right)}<\chi_{n_{1}, t_{1}\left(\rho^{2} / 1-\rho^{2}\right)}^{2}<\frac{n_{1} L C_{1}}{b^{2}\left(1-\rho^{2}\right)}\right] . \\
\operatorname{Pr}\left[\frac{n_{1} L A}{b^{2}\left(1-\rho^{2}\right)}<\chi_{n_{1}, t_{1}\left(\rho^{2} / 1-\rho^{2}\right)}^{2}<\frac{n_{1} L C_{1}}{b^{2}\left(1-\rho^{2}\right)}\right] f\left(t_{1}\right) f\left(t_{2}\right) d t_{1} d t_{2}
\end{gathered}
$$

e

$$
\begin{gathered}
p_{2 b}=\operatorname{Pr}\left[\left(S_{2 x}^{2}<L C_{2}\right) \cap\left(S_{2 y}^{2}<L C_{2}\right) \mid\left(L A<S_{1 x}^{2}<L C_{1}\right) \cap\left(S_{1 y}^{2}<L C_{1}\right)\right] \cdot \operatorname{Pr}\left[\left(L A<S_{1 x}^{2}<L C_{1}\right) \cap\left(S_{1 y}^{2}<L C_{1}\right)\right] \\
p_{2 b}=\int_{n_{1} L A / a^{2}}^{n_{1} L C_{1} / a^{2}} \int_{0}^{n L C_{2} / a^{2}-t_{1}} \operatorname{Pr}\left[\chi_{n,\left(t_{1}+t_{2}\right)\left(\rho^{2} / 1-\rho^{2}\right)}^{2}<\frac{n L C_{2}}{b^{2}\left(1-\rho^{2}\right)} \mid \chi_{n_{1}, t_{1}\left(\rho^{2} / 1-\rho^{2}\right)}^{2}<\frac{n_{1} L C_{1}}{b^{2}\left(1-\rho^{2}\right)}\right] .
\end{gathered}
$$




$$
\begin{gathered}
\operatorname{Pr}\left[\chi_{n_{1}, t_{1}\left(\rho^{2} / 1-\rho^{2}\right)}^{2}<\frac{n_{1} L C_{1}}{b^{2}\left(1-\rho^{2}\right)}\right] f\left(t_{1}\right) f\left(t_{2}\right) d t_{1} d t_{2} \\
\operatorname{com} f\left(t_{i}\right)=\frac{1}{2^{n_{i} / 2} \Gamma\left(n_{i} / 2\right)} e^{-t_{i} / 2} t_{i}^{\left(n_{i} / 2\right)-1} \quad i=1,2 .
\end{gathered}
$$

O limite de controle do segundo estágio, $L C_{2}$, é obtido pela expressão (A4), fazendo $a=b=1$ e $p_{2}=\alpha_{2}$. O valor de $\alpha_{2}$ é especificado pelo usuário.

O poder do gráfico de VMAX com amostragem dupla é dado por:

$$
p=p_{1}+p_{2}
$$

Portanto, $\mathrm{NMA}=1 / \mathrm{p}$.

Para a obtenção do risco $\alpha$ do gráfico de VMAX com amostragem dupla basta fazer $a=b=1$ nas expressões de $p_{1}$ e $p_{2}$. Então, $\alpha=p_{1}+p_{2}$.

\section{APÊNDICE III: GRÁFICO DE |S| COM AMOSTRAGEM DUPLA PROPOSTO POR GRIGORYAN E HE (2005)}

Grigoryan e He (2005) propuseram um gráfico de controle multivariado com amostragem dupla baseado na estatística $|\mathbf{S}|$. Os limites de controle do primeiro estágio são dados por $V_{1}$ e $V_{2}$ e, no segundo estágio, por $V_{3}$.

O esquema de decisão baseado na amostragem dupla é descrito em 5 passos:

Passo 1: Retire a primeira subamostra de tamanho $n_{1}$ e calcule a estatística $Y=\left(\left|\mathbf{S}_{1}\right|-b_{1}\left|\boldsymbol{\Sigma}_{0}\right|\right) / b_{2}^{1 / 2}\left|\boldsymbol{\Sigma}_{0}\right|$, sendo $\mathrm{S}_{1}$ a matriz de covariâncias amostral.

Passo 2: Se $Y$ estiver no intervalo $\left[-V_{1}, V_{1}\right]$ então o gráfico de controle indica que o processo está em controle.

Passo 3: Se $Y$ estiver no intervalo $\left[V_{2}, \infty\right]$ ou $\left[-\infty,-V_{2},\right]$, então o gráfico de controle indica que o processo está fora de controle.

Passo 4: Se $Y$ estiver no intervalo $\left[-V_{2},-V_{1}\right]$ ou $\left[V_{1}, V_{2}\right]$, então retire a subamostra de tamanho $n_{2}$ e calcule a seguinte estatística:

$Y_{1}=\left(\left|\mathbf{S}_{12}\right|-b_{11}\left|\mathbf{\Sigma}_{0}\right|\right) / b_{22}^{1 / 2}\left|\mathbf{\Sigma}_{0}\right|$, sendo $\mathbf{S}_{12}$ a matriz de covariâncias amostral da amostra $n=n_{1}+n_{2}$.

Passo 5: Se $Y_{1}$ estiver no intervalo $\left[-V_{3}, V_{3}\right]$ então o gráfico de controle indica que o processo está em controle; caso contrário, o gráfico de controle sinaliza um desajuste do processo.

As constantes $b_{1}, b_{2}, b_{11}, b_{22}$ dependem de $n_{1}, n_{2}$ e de $p$. Para um $\alpha=0,5 \%$ e uma determinada perturbação na variabilidade do processo, medida por $c^{2}$, Grigoryan e He (2005) determinaram os valores ótimos de $n_{1}$ e $n_{2}$ que minimizam o NMA. Os limites de controle $V_{1}, V_{2}$ e $V_{3}$ foram obtidos por simulação, para um determinado risco $\alpha$. Os valores de

$\alpha_{1}$ e $\alpha_{2}$ não foram apresentados. 